\title{
Identificação de Danos Empregando um Modelo de Dano Contínuo e o Método de Monte Carlo com Cadeias de Markov ${ }^{\dagger}$
}

\author{
J.S. TEIXEIRA, L.T. STUTZ*, A.J. SILVA NETO e D.C. KNUPP
}

Recebido em 31 agosto, 2014 / Aceito em 25 agosto, 2015

\begin{abstract}
RESUMO. O presente trabalho apresenta um estudo referente à aplicação da abordagem Bayesiana como técnica de solução do problema de identificação de danos estruturais, onde a integridade da estrutura é continuamente descrita por um parâmetro denominado parâmetro de coesão. A estrutura escolhida para análise é uma viga simplesmente apoiada do tipo Euler-Bernoulli. A identificação de danos é baseada em alterações na resposta impulsiva da estrutura, provocadas pela presença dos mesmos. O problema direto é resolvido através do Método de Elementos Finitos (MEF), que, por sua vez, é parametrizado pelo parâmetro de coesão da estrutura. O problema de identificação de danos é formulado como um problema inverso, cuja solução, do ponto de vista Bayesiano, é uma distribuição de probabilidade a posteriori do parâmetro de coesão, obtida utilizando-se a metodologia de amostragem de Monte Carlo com Cadeias de Markov. As incertezas inerentes aos dados medidos serão contempladas na função de verossimilhança. São apresentadas três estratégias de solução e um conjunto de resultados numéricos, onde considera-se diferentes níveis de ruído para as três estratégias de solução adotadas.
\end{abstract}

Palavras-chave: identificação de danos, modelo de dano contínuo, inferência Bayesiana, cadeia de Markov.

\section{INTRODUÇÃO}

A avaliação da integridade estrutural é de fundamental importância nas áreas de engenharia mecânica, civil, aeroespacial, petróleo, dentre outras. Uma estrutura danificada pode trazer graves consequências sociais, econômicas e ambientais. Por isso, torna-se imprescindível determinar a segurança dos sistemas e estruturas, atenuando erros e maximizando os níveis de confiabilidade. Para garantir a segurança estrutural, é indispensável o desenvolvimento de metodologias para a identificação de danos.

Muitos são os métodos de identificação de danos estruturais encontrados na literatura, dentre os quais pode-se destacar métodos baseados em respostas no domínio do tempo, apresentado em

\footnotetext{
*Autor correspondente: Leonardo Tavares Stutz.

$\dagger$ Trabalho apresentado no XXXV Congresso Nacional de Matemática Aplicada e Computacional. Instituto Politécnico, IPRJ, UERJ - Universidade do Estado do Rio de Janeiro, 28610-974 Nova Friburgo, RJ, Brasil. E-mails: josyelly1@gmail.com; 1tstutz@iprj.uerj.br; ajsneto@iprj.uerj.br; diegoknupp@iprj.uerj.br
} 
[1], baseados em parâmetros modais, abordado por [2] e em funções de resposta em frequência apresentado por [3], dentre outros.

Neste trabalho, a identificação de danos estruturais é realizada a partir da inferência Bayesiana $[4,5]$. Dentre suas vantagens, pode-se destacar a capacidade de incluir informações a priori, a facilidade de incorporá-las em um contexto formal de decisão, o tratamento explícito das incertezas do problema e a habilidade de assimilar novas informações em contextos adaptativos. Nos últimos anos, esta abordagem tem sido utilizada como ferramenta para resolver diversos problemas de estimação de parâmetros, onde as grandezas do problema são modeladas como variáveis aleatórias.

A seção 2 apresenta a formulação do problema direto, onde é descrito o parâmetro de coesão e a equação que modela o problema. Na seção 3 tem-se a definição do problema de identificação de danos como solução do problema inverso via inferência Bayesiana e a apresentação do método de Monte Carlo via Cadeias de Markov, implementado através do algoritmo de MetropolisHastings. A seção 4 apresenta um conjunto de resultados numéricos, levando-se em conta diferentes níveis de ruído, adicionados aos dados experimentais sintéticos, para as três estratégias adotadas. E na seção 5 apresenta-se as conclusões e sugestões para trabalhos futuros.

\section{FORMULAÇÃO DO PROBLEMA DIRETO}

Na metodologia de identificação de danos adotada, a integridade da estrutura é considerada como sendo continuamente descrita, no domínio do corpo, por um parâmetro estrutural denominado parâmetros de coesão $\boldsymbol{\beta}(x)$ [2]. Este parâmetro está relacionado com a ligação entre os pontos materiais e pode ser interpretado como uma medida do estado de coesão local do material.

Considerando-se que o dano afeta apenas as propriedades elásticas da estrutura, hipótese comumente adotada na literatura, o campo de coesão é definido, em termos da rigidez à flexão, como

$$
\boldsymbol{\beta}(x)=\frac{E(x) I(x)}{E_{0} I_{0}}
$$

onde $E_{0}$ e $I_{0}$ são, respectivamente, os valores nominais do módulo de elasticidade e do momento de inércia de área da seção transversal da viga. Definido deste modo, o campo de coesão é capaz de modelar alterações, devido à presença de danos estruturais, no módulo de elasticidade e/ou no momento de inércia da seção transversal. Se $\boldsymbol{\beta}=1$, considera-se que todas as ligações entre os pontos materiais foram preservadas, ou seja, não há danos na estrutura. Se $\beta=0$, uma ruptura local é considerada, já que todas as ligações entre os pontos materiais foram desfeitas. Para $0<\beta<1$ tem-se a indicação de algum dano na estrutura.

Considerando-se uma viga com seção transversal retangular e módulo de elasticidade uniforme, por simplicidade, o campo de coesão pode ser escrito como

$$
\boldsymbol{\beta}(x)=\left(\frac{h(x)}{h_{0}}\right)^{3}
$$

onde $h(x)$ é a espessura em uma posição genérica $x$ e $h_{0}$ é a espessura nominal da viga. 
O Método de Elementos Finitos (MEF) é empregado para obtenção de uma solução aproximada, fisicamente satisfatória, da equação que modela o problema físico abordado, através do qual obtém-se o seguinte sistema de equações lineares

$$
\mathbf{M} \ddot{\mathbf{u}}+\mathbf{D} \dot{\mathbf{u}}+\mathbf{K}(\boldsymbol{\beta}) \mathbf{u}=\mathbf{f}
$$

onde $\mathbf{u}$ é o vetor de coordenadas generalizadas, $\mathbf{M}$ é a matriz de massa, $\mathbf{D}$ é a matriz de amortecimento, $\mathbf{K}(\boldsymbol{\beta})$ é a matriz de rigidez, f é o vetor de carregamento e $\boldsymbol{\beta}$ é o vetor contendo os valores do parâmetro de coesão nos nós da malha de elementos finitos utilizada para a discretização deste campo.

\section{FORMULAÇÃO DO PROBLEMA INVERSO}

O problema inverso de identificação de danos estruturais é fundamentado no método da máxima verossimilhança, comumente utilizado para resolver problemas de estimação. Para sua construção, adota-se duas hipóteses fundamentais:

(i) Hipótese do modelo perfeito, onde considera-se que o modelo descreve perfeitamente o problema físico estudado. Portanto, os dados experimentais não seguem exatamente as relações impostas pelo modelo devido, exclusivamente, aos inevitáveis erros ocorridos durante os experimentos.

(ii) Hipótese do experimento bem feito, onde considera-se que o experimento é conduzido de tal forma que os erros de medição cometidos durante a execução do experimento sejam mínimos, implicando assim, na máxima probabilidade de se encontrar os dados experimentais [6].

Supondo que a distribuição dos erros experimentais seja conhecida, temos então à disposição a curva de densidade de probabilidade $P\left(\boldsymbol{Z}_{E} ; \boldsymbol{Z}, \boldsymbol{V}\right)$, que descreve a probabilidade de se encontrar os dados experimentais $\boldsymbol{Z}_{E}$, dado a resposta prevista pelo modelo $\boldsymbol{Z}$, e uma medida de variância dos erros experimentais $\boldsymbol{V}$.

Considerando-se que os erros experimentais apresentam uma distribuição de probabilidade normal (gaussiana), a verossimilhança é dada por [6]

$$
P\left(\boldsymbol{Z}_{E} ; \boldsymbol{Z}, \boldsymbol{V}\right)=\frac{1}{\sqrt{\operatorname{det} \boldsymbol{V}}} \frac{1}{\sqrt{(2 \pi)^{n_{e}}}} \exp \left[-\frac{1}{2}\left(\boldsymbol{Z}_{E}-\boldsymbol{Z}\right)^{T} \boldsymbol{V}^{-1}\left(\boldsymbol{Z}_{E}-\boldsymbol{Z}\right)\right]
$$

onde $n_{e}$ é o número de dados experimentais utilizados no problema.

Obtém-se como consequência direta das hipóteses do modelo perfeito e experimento bem feito a definição do problema de estimação de parâmetros, cujo objetivo é maximizar a função $P\left(\boldsymbol{Z}_{E} ; \boldsymbol{Z}, \boldsymbol{V}\right)$, ou seja, maximizar a probabilidade de encontrar os dados experimentais, segundo a distribuição de probabilidade, já conhecida, dos erros experimentais. Portanto, o problema inverso de identificação de danos pode ser definido como

$$
\max _{\boldsymbol{\beta}} P\left(\boldsymbol{Z}_{E} ; \boldsymbol{Z}, \boldsymbol{V}\right)
$$




\subsection{Inferência Bayesiana}

Do ponto de vista Bayesiano, a solução do problema inverso, dadas as observações experimentais a posteriori $\boldsymbol{Z}_{E}$, é uma função de densidade de probabilidade de $\boldsymbol{\beta}$, denotada por $P_{\text {post }}\left(\boldsymbol{\beta} \mid \boldsymbol{Z}_{E}\right)$, que pode ser escrita, de acordo com a fórmula do teorema de Bayes, como

$$
P_{\text {post }}\left(\boldsymbol{\beta} \mid \boldsymbol{Z}_{E}\right)=\frac{P\left(\boldsymbol{Z}_{E} \mid \boldsymbol{\beta}\right) P_{p r}(\boldsymbol{\beta})}{P\left(\boldsymbol{Z}_{E}\right)}
$$

onde $P\left(\boldsymbol{Z}_{E} \mid \boldsymbol{\beta}\right)$, com as hipóteses do modelo perfeito e de experimento bem feito e quando as flutuações das medidas experimentais seguem a distribuição normal, é a verossimilhança e $P\left(\boldsymbol{Z}_{E}\right)$ é a densidade marginal, que funciona como uma constante de normalização.

Quando a distribuição a posteriori pode ser obtida através de expressão analítica ou simulação numérica, a solução do problema segue diretamente do Teorema de Bayes. Caso contrário, sua obtenção torna-se muito complicada ou impossível. Nestes casos, as técnicas de amostragem são muito utilizadas, onde busca-se a simulação de amostras da distribuição de interesse $P_{\text {post }}\left(\boldsymbol{\beta} \mid \boldsymbol{Z}_{E}\right)$ para então serem feitas inferências dessa distribuição de probabilidade, como a obtenção de momentos estatísticos de média e desvio padrão.

\subsection{Método de Monte Carlo via Cadeias de Markov}

Amostras da distribuição a posteriori de interesse, cuja simulação direta é inviável, podem ser obtidas através dos métodos de Monte Carlo via Cadeias de Markov (MCMC). A ideia geral é simular amostras aleatórias no domínio do parâmetro $\boldsymbol{\beta}$, de tal forma, que convirjam para a distribuição a posteriori $P_{\text {post }}\left(\boldsymbol{\beta} \mid \boldsymbol{Z}_{E}\right)$, utilizando técnicas de simulação iterativa, baseadas em cadeias Markov.

Uma cadeia de Markov é um processo estocástico tal que, para uma sequência $\left(\beta_{0}, \ldots, \beta_{i}, \ldots\right)$, a distribuição de $\beta_{i}$ depende exclusivamente do valor anterior $\beta_{i-1}$. Desse modo, para qualquer subconjunto $A$, tem-se

$$
P\left(\beta_{i} \in A \mid \beta_{0}, \ldots, \beta_{i-1}\right)=P\left(\beta_{i} \in A \mid \beta_{i-1}\right)
$$

Para a obtenção das cadeias de Markov são utilizados algoritmos específicos. Neste trabalho foi utilizado o algoritmo de Metropolis-Hastings, que faz uso de uma função densidade de probabilidade auxiliar $q$, da qual seja fácil se obter valores amostrais. $\mathrm{O}$ algoritmo pode ser especificado pelos seguintes passos:

Passo 1: Iniciar a cadeia com o estado inicial $\boldsymbol{\beta}_{0}$.

Passo 2: Selecionar um candidato $\boldsymbol{\beta}^{*}$ a partir do estado atual $\boldsymbol{\beta}_{i-1}$, usando a distribuição auxiliar $\mathbf{q}\left(\boldsymbol{\beta}^{*} \mid \boldsymbol{\beta}_{i-1}\right)$.

Passo 3: Calcular o fator de aceitação $\gamma$, dado pela Razão de Hastings

$$
\gamma=\min \left[1, \frac{P\left(\beta^{*}\right) q\left(\beta^{*} \mid \beta_{i-1}\right)}{P\left(\beta_{i-1}\right) q\left(\beta_{i-1} \mid \beta^{*}\right)}\right] .
$$


Passo 4: Calcular um número aleatório $U$ vindo de uma distribuição uniforme entre 0 e 1.

Passo 5: Se $U \leq \gamma$ então aceita-se o novo valor e faz-se $\boldsymbol{\beta}_{i}=\boldsymbol{\beta}^{*}$, caso contrário, rejeita-se $\boldsymbol{\beta}^{*}$ e faz-se $\boldsymbol{\beta}_{i}=\boldsymbol{\beta}_{i-1}$.

Passo 6: Incrementar o contador $i$ para $i+1$ e voltar ao passo 2, a fim de gerar a cadeia de Markov $\left\{\boldsymbol{\beta}_{1}, \boldsymbol{\beta}_{2}, \cdots, \boldsymbol{\beta}_{N}\right\}$, onde $N$ é o tamanho da cadeia.

Os estados gerados até que se alcance o equilíbrio são chamados de amostras de aquecimento (burn-in) [7]. Estas são descartadas para que possamos realizar a inferência estatística dos parâmetros de coesão.

No presente trabalho, $q$ é definida como uma distribuição de probabilidade normal, nesse caso, $q$ é simétrica, ou seja, $q\left(\beta^{*} \mid \beta_{i-1}\right)=q\left(\beta_{i-1} \mid \beta^{*}\right)$. Logo, o Passo 3 é simplificado e a Equação (1) pode ser reescrita como

$$
\gamma=\min \left[1, \frac{P\left(\beta^{*}\right)}{P\left(\beta_{i-1}\right)}\right]
$$

onde $P\left(\beta^{*}\right)$ e $P\left(\beta_{i-1}\right)$ podem ser diretamente calculadas.

\section{RESULTADOS}

Neste trabalho, considerou-se uma viga bidimensional de Euler-Bernoulli de aço e simplesmente apoiada. As simulações foram realizadas com uma discretização espacial da viga em 24 elementos do tipo Euler-Bernoulli. As aproximações do campo de deslocamentos e do campo de coesão são realizadas utilizando-se a mesma malha de elementos finitos.

A imposição do dano à viga é realizada através de uma redução na altura relativa da seção transversal $h(x) / h_{0}=\sqrt[3]{\boldsymbol{\beta}(x)}$, nos nós contidos no interior das regiões danificadas. Os dados experimentais são obtidos a partir da resposta impulsiva do sistema, dada pelo MEF, para um valor prescrito de $\boldsymbol{\beta}$. O ruído, adicionado aos dados experimentais, é definido indiretamente pela razão sinal ruído, que é dada por $S N R=10 \log \left(P_{S} / P_{n}\right)$, onde $P_{S}$ e $P_{n}$ correspondem à potência do sinal $s$ e à potência do ruído $n$, respectivamente.

Para a obtenção dos dados utilizados no processo de identificação de danos estruturais, considera-se uma excitação impulsiva ou impulso de Dirac em $x=0,2433 \mathrm{~m}$ e que a resposta impulsiva, em deslocamento, foi medida na mesma posição. Os danos foram impostos na estrutura nas posições $x=0,5475 \mathrm{~m}$ e $x=1,2775 \mathrm{~m}$, que correspondem, respectivamente, aos nós 10 e 22 da malha de elementos finitos. Foram impostos danos de mesma intensidade, com $h(0,5475) / h_{0}=h(1,2775) / h_{0}=0,9$, que correspondem a $\boldsymbol{\beta}_{10}=\boldsymbol{\beta}_{22}=0,729$.

Três estratégias de solução foram utilizadas no processo de identificação de danos estruturais. Na Estratégia 1, considera-se que a distribuição de probabilidade auxiliar, utilizada no algoritmo de Metropolis-Hastings para amostrar valores dos parâmetros de coesão, tem o mesmo desvio padrão. Na Estratégia 2, considera-se que a distribuição tem desvios diferenciados, determinados a partir de uma análise inicial do processo de identificação de danos, onde aos parâmetros associados às regiões em torno daquelas identificadas como potencialmente danificadas recebem 
desvios diferenciados dos demais parâmetros. Nas estratégias 1 e 2, todos os parâmetros são atualizados a cada iteração. Na Estratégia 3, considera-se que após uma análise inicial do processo de identificação de danos, apenas os parâmetros em torno das regiões identificadas como potencialmente danificadas são atualizados e os demais parâmetros permanecem iguais a 1, ou seja, sem dano.

No trabalho [8], apenas a Estratégia 2 é utilizada como técnica de solução do problema de identificação de danos, onde somente o parâmetro associado à região identificada como potencialmente danificada recebe um desvio diferenciado. No presente trabalho, assim como em [9], além de atribuir um desvio diferenciado para os parâmetros associados às regiões potencialmente danificadas, atribui-se também o mesmo desvio para os parâmetros associados a uma pequena região em torno delas, além da utilização das Estratégias 1 e 3.

Analisaremos dois casos onde as três estratégias propostas serão aplicadas utilizando dados experimentais corrompidos por um ruído de $30 \mathrm{~dB}$, Caso 1, e $20 \mathrm{~dB}$, Caso 2.

A seguir são apresentados os resultados da identificação de danos para o Caso 1, onde foram consideradas cadeias de Markov com 100.000 estados para a Estratégia 1 e 20.000 estados para as Estratégias 2 e 3.

A partir de uma análise qualitativa verifica-se que, para o ruído adotado ( $\mathrm{SNR}=30 \mathrm{~dB}$ ), todas as estratégias foram capazes de localizar com acurácia os danos existentes na estrutura. No entanto, da Figura 1a, observa-se que a Estratégia 1 indicou a existência de pequenos danos em regiões intactas da estrutura e um maior intervalo para um grau de confiança de 99\%, quando comparado com os resultados obtidos pelas Estratégias 2 e 3, Figuras 1c e 1e, respectivamente. Pode-se observar ainda a lenta convergência da cadeia obtida para o parâmetro $\boldsymbol{\beta}_{10}$ através da Estratégia 1, Figura 1b, e a rápida convergência das cadeias para as Estratégias 2 e 3, Figuras 1d e 1f, respectivamente.

A seguir são apresentados os resultados da identificação de danos para o Caso 2, onde, devido à utilização de um ruído muito intenso $(\mathrm{SNR}=20 \mathrm{~dB})$, foram consideradas cadeias de Markov com 100.000 estados para todas as estratégias.

Observa-se que o resultado obtido pela Estratégia 1, Figura 2a, indicou danos em regiões intactas da estrutura e, nas regiões realmente danificadas, obteve-se intensidades consideravelmente menores do que as reais intensidades, além de apresentar uma cadeia de Markov não convergida, como pode ser visto na Figura 2b. As Estratégias 2 e 3, Figuras 2c e 2e, respectivamente, apresentaram resultados satisfatórios, apresentando cadeias de Markov convergidas e bem misturadas, Figuras 2d e 2f. O resultado obtido pela Estratégia 3 indicou acuradamente as regiões danificadas, porém, observou-se danos com intensidades menores e levemente distribuídos em pequenas regiões em torno dos danos reais. Enquanto que, o resultado obtido pela Estratégia 2, indicou adequadamente as regiões danificadas, porém, apenas o primeiro dano foi acuradamente descrito. O segundo dano apresentou-se mais distribuído e observou-se ainda uma diferença mais acentuada entre a intensidade identificada e a exata. 


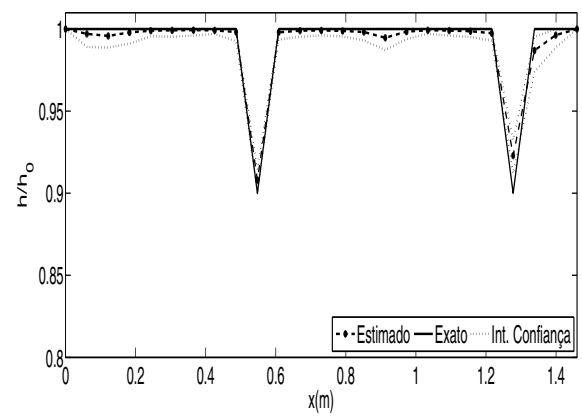

(a) Estratégia 1

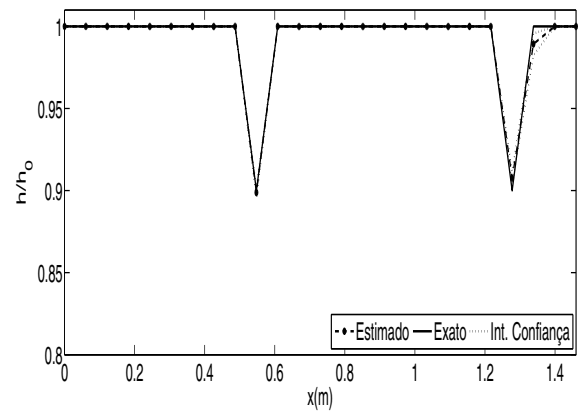

(c) Estratégia 2

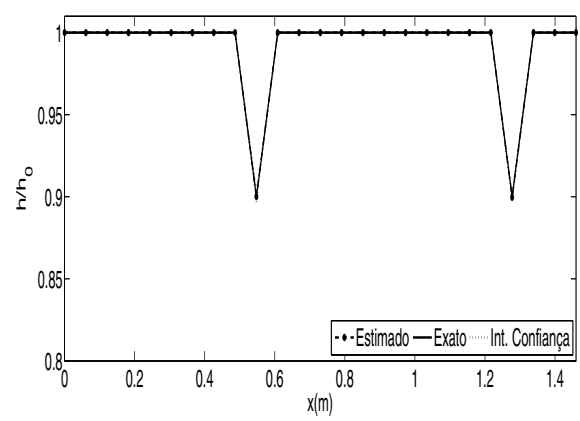

(e) Estratégia 3

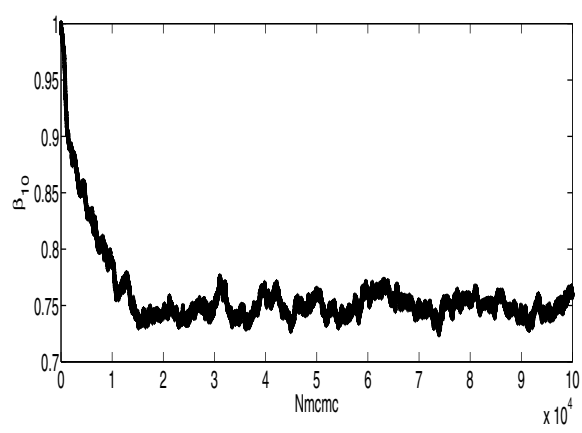

(b) Evolução da Cadeia de Markov para $\boldsymbol{\beta}_{10}$

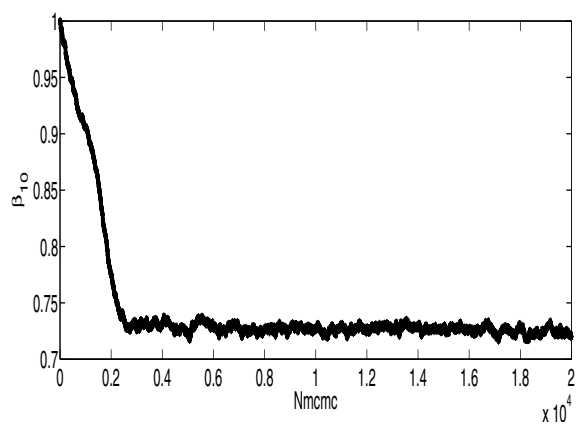

(d) Evolução da Cadeia de Markov para $\boldsymbol{\beta}_{10}$

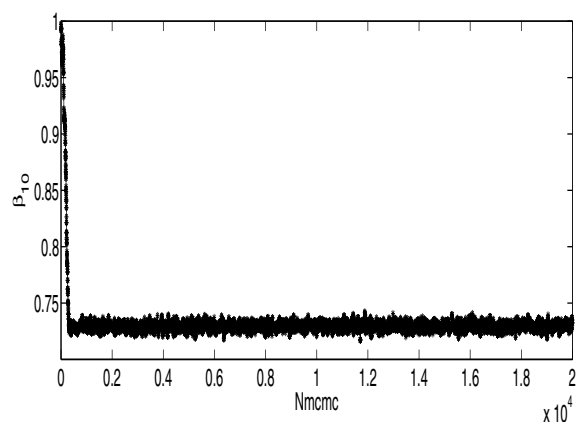

(f) Evolução da Cadeia de Markov para $\boldsymbol{\beta}_{10}$

Figura 1: Resultado da identificação de danos para o Caso 1.

\section{CONCLUSÕES}

O presente trabalho teve como objetivo principal a aplicação da abordagem Bayesiana na identificação de danos estruturais. Apresentou-se uma formulação do problema direto, cuja solução foi obtida através do Método de Elementos Finitos (MEF). A presença do dano estrutural foi modelada a partir de um modelo de dano contínuo, através do parâmetro de coesão estrutural 


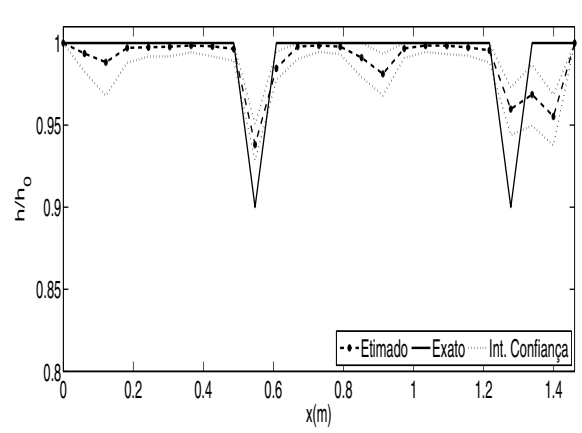

(a) Estratégia 1

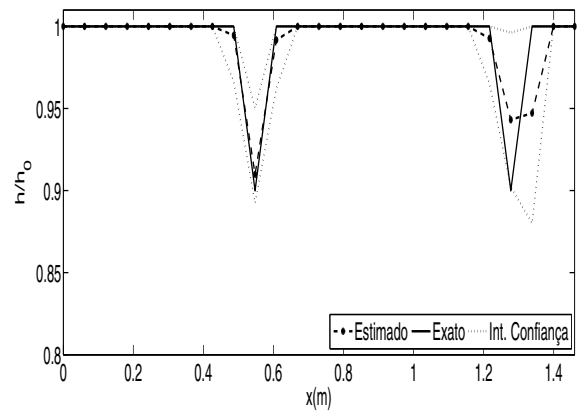

(c) Estratégia 2

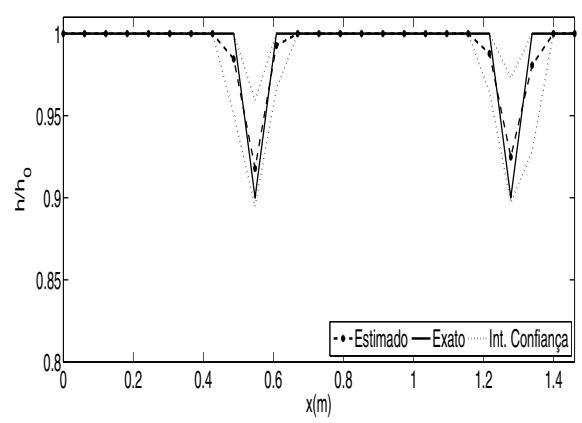

(e) Estratégia 3

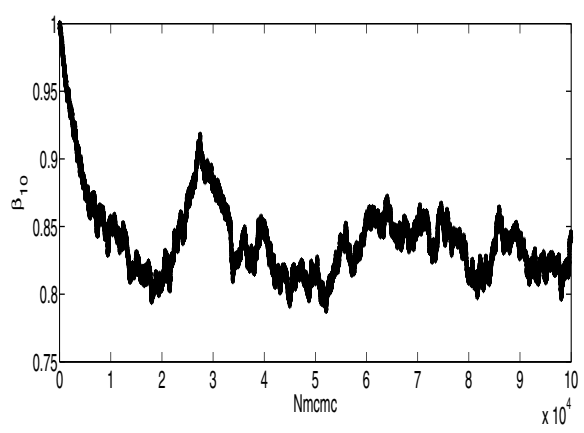

(b) Evolução da Cadeia de Markov para $\boldsymbol{\beta}_{10}$

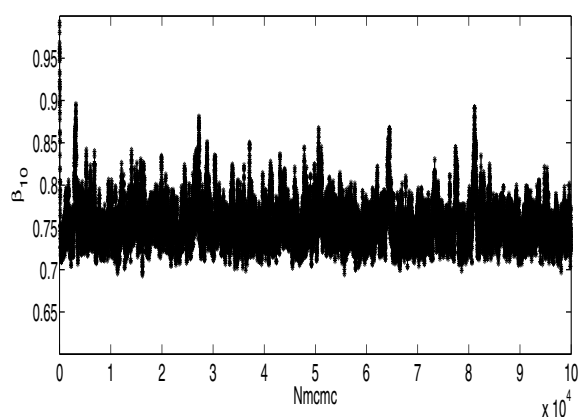

(d) Evolução da Cadeia de Markov para $\boldsymbol{\beta}_{10}$

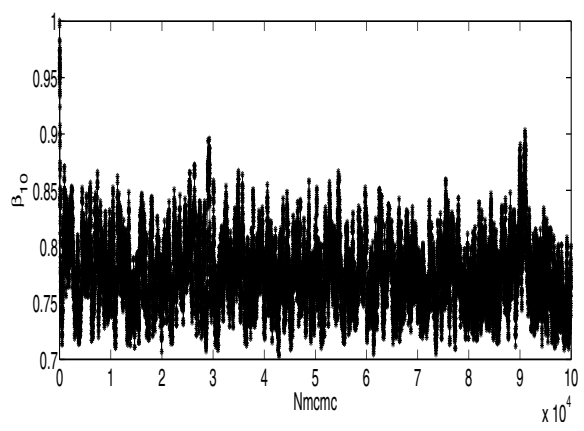

(f) Evolução da Cadeia de Markov para $\boldsymbol{\beta}_{10}$

Figura 2: Resultado da identificação de danos para o Caso 2.

$(\boldsymbol{\beta})$. A solução do problema inverso de estimação foi obtida pelo Método de Monte Carlo com Cadeias de Markov (MCMC), implementado através do algoritmo de Metropolis-Hastings.

Três estratégias de solução foram abordadas, de onde observou-se que as Estratégias 2 e 3 apresentaram melhores resultados em relação à Estratégia 1, demonstrando assim a grande influência que o desvio padrão da distribuição de probabilidade auxiliar tem no processo de identificação de danos. Em relação à quantidade de parâmetros a serem estimados, observou-se que apesar 
de a Estratégia 2 ter apresentado bons resultados para a identificação, a Estratégia 3, apresentou cadeias mais bem misturadas e convergência mais rápida das mesmas. No entanto, a eficácia das Estratégias 2 e 3 está diretamente ligada à identificação acurada da localização das regiões potencialmente danificadas.

Com relação à influência de dados ruidosos na identificação de danos, observou-se que a utilização de dados muito corrompidos por ruídos prejudicam a identificação exata das regiões realmente danificadas e a quantificação acurada das intensidades dos danos reais.

Como sugestões para trabalhos futuros propõe-se o estudo de diferentes distribuições auxiliares como, por exemplo, a distribuição uniforme ou exponencial, para obtenção das cadeias de Markov; a consideração de estruturas mais complexas, como uma placa; utilização de informação a priori informativa para o parâmetro de coesão; e a utilização de outras técnicas de localização de danos, que sejam capazes de localizar acuradamente as regiões danificadas, principalmente quando utiliza-se dados muito corrompidos por ruído.

\title{
AGRADECIMENTOS
}

Os autores agradecem o apoio financeiro da FAPERJ - Fundação Carlos Chagas Filho de Amparo à Pesquisa do Estado do Rio de Janeiro, do CNPq - Conselho Nacional de Desenvolvimento Científico e Tecnológico e da CAPES - Coordenação de Aperfeiçoamento de Pessoal de Nível Superior.

\begin{abstract}
This work presents a study on the application of Bayesian approach as a technique for solving the inverse problem of structural damage identification, where the integrity of the structure is continuously described by a structural cohesion parameter. The structure chosen for analysis is a simply supported Euler - Bernoulli beam. The damage identification is based on changes in the impulse response of the structure caused by the presence. The direct problem is solved by the finite element method (FEM), which, in turn, is parameterized by the cohesion parameter of the structure. The problem of identifying damages is formulated as an inverse problem, whose solution, from the Bayesian framework, is a posteriori probability distribution of the cohesion parameter, obtained using the sampling methodology of Monte Carlo with Markov Chain. The uncertainties inherent to the measured data will be included in the likelihood function. Three solution strategies and a set of numerical results are presented taking into account different noise levels for the three strategies considered.
\end{abstract}

Keywords: Damage identification, Continuum damage model, Bayesian inference, Markov Chain Monte Carlo methods. 


\section{REFERÊNCIAS}

[1] D.A. Castello, L.T. Stutz \& F.A. Rochinha. A time domain technique for defect identification based on a continuous damage model. "Proceedings of the 2002 ASME International Mechanical Engineering Congress \& Exposition”, New Orleans (2002).

[2] L.T. Stutz, D.A. Castello \& F.A. Rochinha. A flexibility-based continuum damage identification approach. Journal of Sound and Vibration, 279 (2005), 641-667.

[3] A. Furukawa, H. Otsuka \& J. Kiyono. Structural damage detection method using uncertain frequency response functions. Journal of Computer-Aided Civil and Infrastructure Engineering, 21 (2006), 292-305.

[4] M. Paez \& D. Gamerman. Bayesian Approaches for the Solution of Inverse Problems. H.R.B. Orlande, O. Fudym, D. Maillet \& R.M. Cotta, Editors, Thermal Measurements and Inverse Techniques, CRC Press, Boca Raton (2011).

[5] J.M. Nichols, E.Z. Moore \& K.D. Murphy, Bayesian identification of a cracked plate using a population-based Markov Chain Monte Carlo method. Journal of Computers and Structures, 89 (2011), 1323-1332.

[6] M. Schwaab \& J.C. Pinto. "Análise de Dados Experimentais I: Fundamentos de Estatística e Estimação de Parâmetros”, E-papers, Rio de Janeiro (2007).

[7] J.P. Kaipio \& E. Somersalo. "Statistical and Computational Inverse Problems". Spring-Verlag, New York (2004).

[8] J.S. Teixeira, D.C. Knupp, L.T. Stutz \& A.J. Silva Neto. Identificação de danos estruturais via método de Monte Carlo com cadeia de Markov, em "Anais do XVI Encontro de Modelagem Computacional”, (2013).

[9] J.S. Teixeira, D.C. Knupp, L.T. Stutz \& A.J. Silva Neto. Application of a Continuum Damage Identification Approach via Markov Chain Monte Carlo Method using the Impulsive Response Function, em "8th International Conference on Inverse Problems in Engineering”, Cracow, Poland (2014). 